\section{European regulatory framework for the clinical translation of bioprinted scaffolds and tissues}

Licia Di Pietro, ${ }^{1}$ Alice Ravizza, 2 Giovanni Vozzi, ${ }^{1}$ Andrés Diaz Lantada, ${ }^{3}$ Arti Ahluwalia, ${ }^{1}$ Carmelo De Maria ${ }^{1}$

1Research Center E. Piaggio and

Department of Information Engineering, University of Pisa, Pisa, Italy;

2Department of Mechanical and

Aerospace Engineering, Politecnico di

Torino, Turin, Italy; ${ }^{3}$ Universidad

Politécnica de Madrid, Madrid, Spain

\begin{abstract}
Tissue Engineering and Regenerative medicine, empowered by Biofabrication technologies, hold the premises to provide solutions to unmet clinical needs, such as organ donor shortage or genetic diseases. These huge advancements are determining a changing scenario, with a quite confusing understanding about the steps toward the clinical translation of new researches and products, giving as result an overestimation or an underestimation of the required in vitro and in vivo tests for their validation. The proper definition and classification of the research products can be considered an action toward the refinement of animal experiments. An appropriate classification is crucial because the complications due to the combination of biological and non-biological materials need the application of specific rules. This paper aims at helping the academic and industrial community to clarify the identification and classification of their research products.
\end{abstract}

\section{Introduction}

Tissue Engineering and Regenerative medicine, empowered by Biofabrication technologies, hold the premises to provide solutions to unmet clinical needs, such as organ donor shortage or genetic diseases. ${ }^{1}$ Bioprinting scaffolds or other implants are changing not only the surgical procedures, but also the organization of hospitals, which can potentially produce in-house these products. ${ }^{2}$ In this context, new biomaterials but also bioprinters and bioreactors are reaching the market. Figure 1 shows the several areas in the bioprinting healthcare scenario.

These huge advancements are determin- ing a changing scenario, with a quite confusing understanding about the steps toward the clinical translation of new researches and products, giving as result an overestimation or an underestimation of the required in vitro and in vivo test for their validation.

\section{Materials and Methods}

The proper definition and classification of the research products can be considered an action toward the refinement of animal experiment. For example, generic term "scaffold" is used to indicate an implantable substrate, which can be correctly classified as Medical Device (MD) or as Advanced Therapy Medicinal Product (ATMP) according to its working principles and/or components. Similarly, bioprinters are normally used to fabricate scaffolds or in vitro model for biological experiment, but they could potentially be used to bioprint in situ biomaterial inks and bionks: in this case, they have to be considered as MD, with the proper request of safety and efficacy. At European level, these requirements are defined by the EU Regulation 2017/745 and $1394 / 2007$, which set the legislative framework for the definition and validation toward commercialization of MDs and ATMPs, respectively.3,4

\section{Results}

With the aim at helping the academic and industrial community to clarify the identification and classification of their research products, Figure 2 tries to clarify these differences with practical examples.
Correspondence: Licia Di Pietro, Research Center E. Piaggio and Department of Information Engineering, University of Pisa, Pisa, Italy.

E-mail: dipietrolicia@gmail.com; licia.dipietro@ing.unipi.it

Key words: Bioprinting; 3D printing; regulation; ATMP; medical device.

Conference presentation: this paper was presented at the Second Centro 3R Annual Meeting - 3Rs in Italian Universities, 2019, June 20-21, University of Genoa, Italy.

Received for publication: 28 October 2019. Accepted for publication: 11 November 2019

This work is licensed under a Creative Commons Attribution NonCommercial 4.0 License (CC BY-NC 4.0).

(C) Copyright: the Author(s), 2019

Licensee PAGEPress, Italy

Biomedical Science and Engineering 2019; 3(s3):108 doi:10.4081/bse.2019.108

\section{Conclusions}

Bioprinting raises questions about the exact legal nature and specific classification of bioprinted-related products. An appropriate classification is crucial, in particular, because the complications due to the combination of biological and non-biological materials need the application of specific rules. This suggests that the additive manufacturing technologies applied to bioprinting need an appropriate legal framework in particular in the domain of pharmaceutical, medical devices, advances therapies, tissues and cells where significant regulatory and socio-ethical challenges are faced.

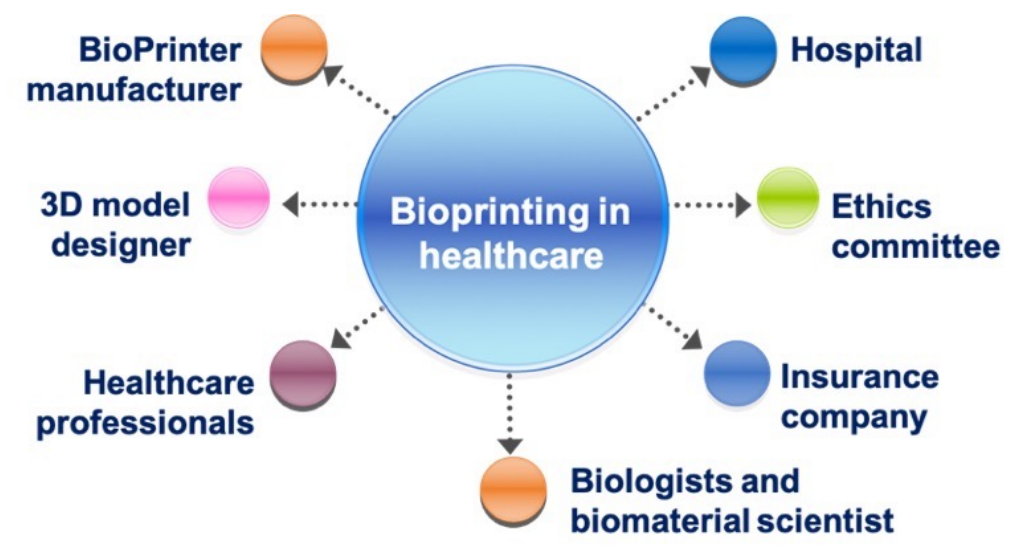

Figure 1. Bioprinting in healthcare. 


\section{Bioprinting products}

in healthcare

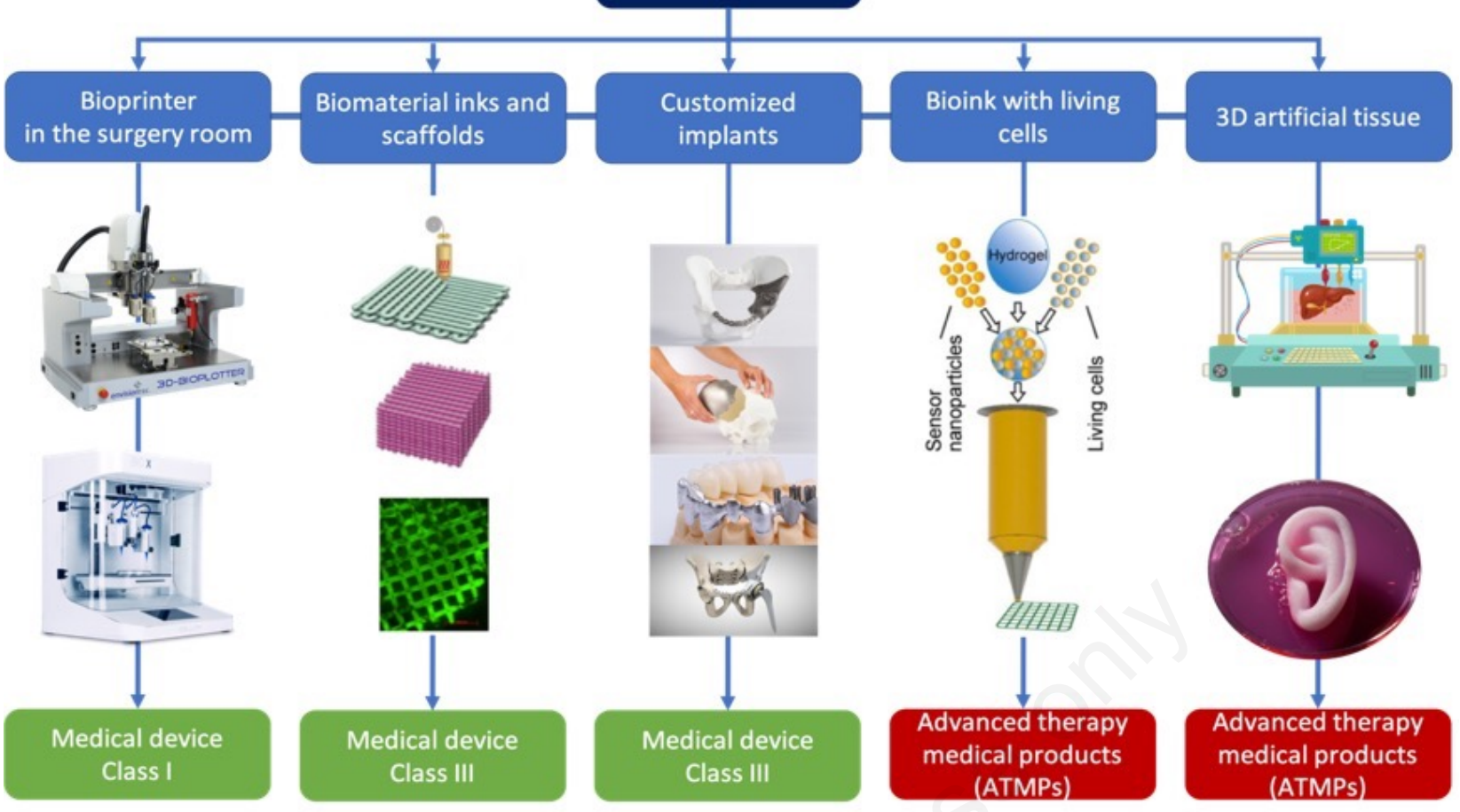

Figure 2. Bioprinting products in healthcare.

\section{References}

1. Gomes ME, Rodrigues MT, Domingues RM, Reis RL. Tissue engineering and regenerative medicine: new trends and directions - a year in review. Tissue Eng Part B Rev 2017;23:211-24.
2. Bishop ES, Mostafa S, Pakvasa M, et al. 3-D bioprinting technologies in tissue engineering and regenerative medicine: Current and future trends. Genes Dis 2017;4:185-95.

3. Regulation (EU) No 2017/745. Available from: https://eur-lex.europa. eu/legal-content /EN/ TXT/?qid=
1557954879210\&uri=CELEX:32017R 0745. Accessed: May 2019.

4. Regulation (EC) No 1394/2007. Available from: https://eur-lex.europa. eu/legal-content/EN/ALL/? uri= CELEX\%3A32007R1394. Accesssed: May 2019. 